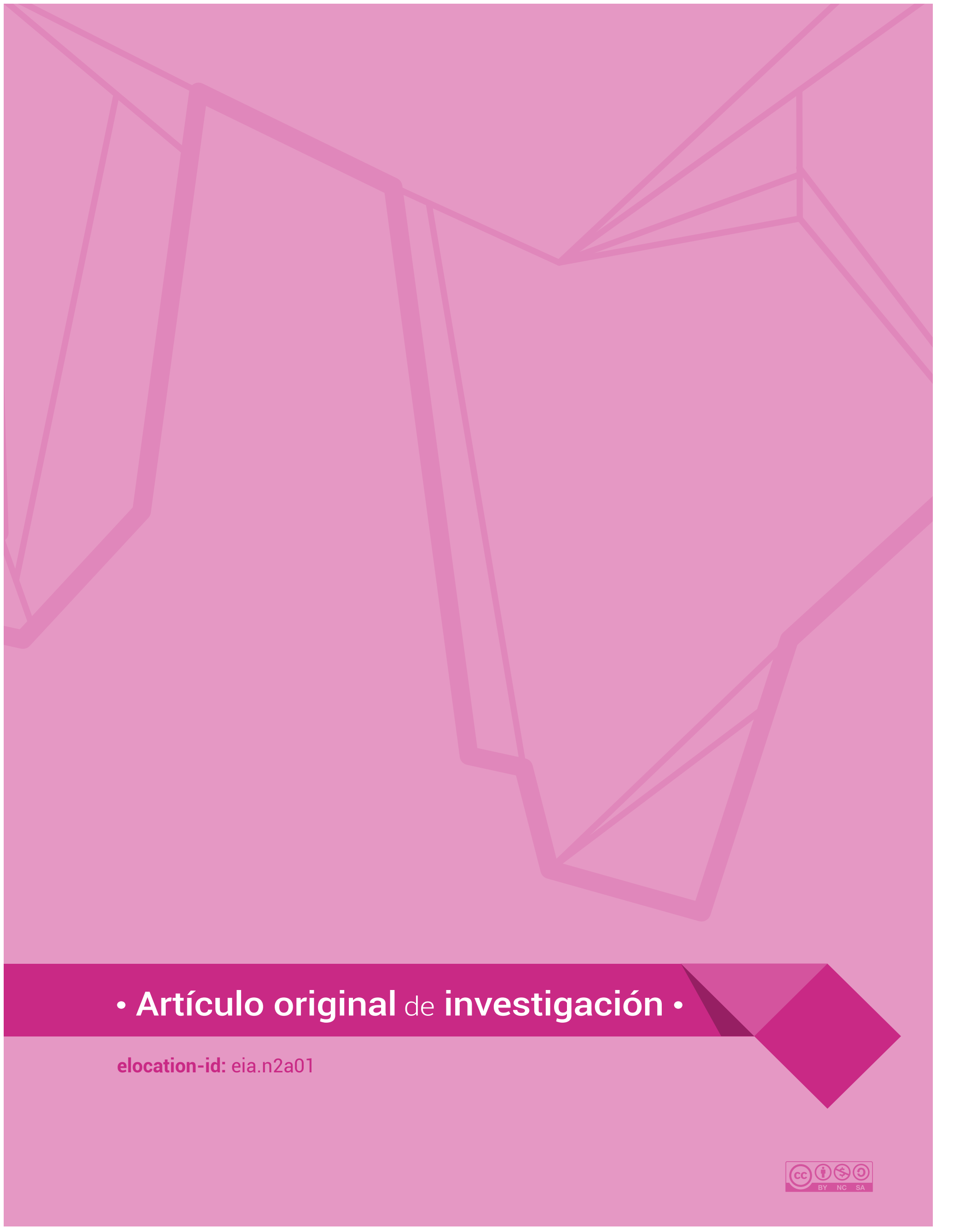




\title{
Prevalencia y causas de la automedicación de anticonceptivos orales en las estudiantes de la Facultad de Enfermería de la Corporación Universitaria Remington
}

\author{
Prevalence and causes of self-medication \\ of oral contraceptives among students \\ of the Faculty of Nursing of Corporación \\ Universitaria Remington
}

Mary L. Hurtado-Perea'; Diana Cárdenas-Valderrama'; Jhonny J. Mosquera-Torres ${ }^{7}$; Alejandra M. Román-Mejía', Isaura Pilar

Sánchez ${ }^{2}$

1 Estudiante de Enfermería, Facultad de Ciencias de la Salud, Corporación Universitaria Remington, Medellín, Colombia

2 Docente-Investigador, Facultad de Ciencias de la Salud, Grupo de Investigaciones Biomédicas, Corporación Universitaria Remington, Medellín, Colombia

\section{Resumen}

Las investigaciones sobre automedicación en el personal universitario han cobrado gran importancia debido a su crecimiento y al problema de salud pública que representa. El objetivo de este trabajo fue identificar la prevalencia de automedicación con anticonceptivos orales y las causas que conllevan a esta práctica en las estudiantes de la Facultad de Enfermería de la Corporación Universitaria Remington. Se diseñó una encuesta para identificar las causas de automedicación con anticonceptivos en 230 estudiantes, efectos secundarios asociados a su consumo y medicamentos más utilizados. Se confirmó la práctica de la automedicación con anticonceptivos orales en $43.5 \%$. La prevalencia asociada a los grupos de edad fueron 17-21 años (36 \%), 22-26 (37\%) 27-31(20\%) y de 32-36 (7\%). La causa de automedicación más frecuente fue la buena referencia del fármaco en $61 \%$. Los medicamentos más usados fueron Microgynon (19\%), Yax (17\%) y Diane 35 (17\%). El efecto secundario que se asoció al uso de estos medicamentos fue la cefalea (61 \%) con Mycrogynon, (41 \%) Yax y (41 \%) Diane 35. Los hallazgos del presente trabajo son de importancia para nuestra comunidad universitaria y como enfermeros profesionales en formación, evidenciándose alta frecuencia de automedicación de anticonceptivos orales en las estudiantes. Es propicio generar conciencia sobre lo importante de una asesoría correcta que 
cumpla los parámetros de seguridad y efectividad con respecto al uso de los anticonceptivos orales.

Palabras claves: antecedentes personales, anticonceptivos, automedicación efectos secundarios, prescripción, signos y síntomas.

\section{Abstract}

Research on self-medication among university students has become extremely important since it is a growing problem and an increasing public health concern. This paper aims to identify the prevalence of self-medication of oral contraceptives as well as the causes leading to this practice among students of the Faculty of Nursing of Corporación Universitaria Remington. A survey was conducted to identify the causes of self-medication of contraceptives in a sample of 230 students, the side effects associated with their consumption and the most commonly used medicines. The practice of self-medication of oral contraceptives was confirmed in $43.5 \%$ of the population. The prevalence associated with age groups was calculated as follows: 17-21 years (36\%), 22-26 (37\%) 27-31 (20\%) and 32-36 (7\%). The most frequent cause of self-medication was a positive reference of the drug (61\%). The most widely used medications were Microgynon (19\%), Yax (17\%) and Diane 35 (17\%). The side effect associated with the use of these medications was a headache (61\%) with Mycrogynon, (41\%) Yax and (41\%) Diane 35. The findings are relevant both for our university community and for the authors as professional nurse trainees. These results show a high frequency of self-medication of oral contraceptives among the institution's students. Awareness should be raised about the importance of proper counseling that meets the safety and effectiveness standards regarding the use of oral contraceptives.

Keywords: personal background, contraceptives, self-medication side effects, prescription, signs and symptoms.

\section{Introducción}

La automedicación es una práctica que ha trascendido a lo largo de la historia del hombre, que busca aliviar o prevenir algún síntoma por medio del uso de medicamentos sin una prescripción por personal de salud requerido. En la actualidad este tópico ha sido tema de investigación, debido a su crecimiento y al problema de salud pública que representa, ya que en muchos casos se ve afectada la salud integral de las personas por acudir a este método tradicional (Álvarez Gómez, 2013). Las investigaciones sobre la automedicación en el personal universitario han cobrado gran importancia, ya que representa de alguna manera una problemática de salud debido a los riegos que acarrea el consumo de medicamentos de manera autoritaria.

En este contexto, estudios han demostrado que las mujeres universitarias son las que mayormente usan la práctica de automedicarse. Según lo reportado en un estudio en Colombia, el uso de anticonceptivos orales automedicados representa una alta prevalencia que indica que el $22.5 \%$ de las usuarias incurrieron en esta práctica durante el desarrollo del estudio y el $24.7 \%$ estaban usando o usaron el método anticonceptivo hormonal por autoprescripción durante el tiempo de la investigación (Hincapié García, Quintero Agudelo, Gaviria, Estupiñán Cabrera, y Amariles, 2013). Otro estudio demostró que la elección inicial del método fue la píldora en $57 \%$ de los casos, $15 \%$ el anillo vaginal y $13 \%$ el parche transdérmico. Sin embargo, es importante resaltar en este estudio que luego de la asesoría educativa en relación con la elección del método, los porcentajes variaron incluyendo $38 \%$ la píldora, $33 \%$ el anillo vaginal, y $19 \%$ el parche transdérmico (Cáceres chú, Thornberry, Má Cárdenas, y Ramos Castillo, 2014). Entre los grupos de 
medicamentos más consumidos por estudiantes universitarias sin una prescripción médica, se incluyen antibióticos, sedantes, ansiolíticos, antihistamínicos, analgésicos, anticonceptivos orales y broncodilatadores; sin tener en cuenta posibles efectos secundarios que pueden aparecer por el uso de estos medicamentos (Arrate Negret, Linares Despaigne, Molina Hechavarría, Sánchez Imbertl, y Arias Carbonell, 2013).

Así mismo, estos estudios han mostrado la posible relación de los fármacos anticonceptivos orales con la aparición de enfermedades que ponen en riesgo la salud de las mujeres que los usan (Arrate Negret, et al., 2013). Todos estos hallazgos nos motivan a plantear un estudio que permita establecer cuál es la prevalencia de la automedicación con anticonceptivos orales y cuáles son las causas que conllevan a esta práctica a las estudiantes de la Facultad de Enfermería de la Corporación Universitaria Remington que cursan del primer al octavo semestre del periodo II de 2015.

\section{Materiales y métodos}

\section{Tipo de estudio}

Este es un estudio descriptivo, donde la población total fue de 353 estudiantes, de manera aleatoria simple se llevaron a cabo 230 encuestas de las cuales 100 cumplieron con los criterios de inclusión, (estudiantes femeninas de la Facultad de Enfermería, uso de anticonceptivos orales automedicados) quienes decidieron participar de manera autónoma y voluntaria en el estudio.

\section{Métodos}

Para la recolección de datos se utilizó una encuesta que contenía 11 preguntas y se aplicó de manera escrita. En el instrumento se consideraron algunas variables de acuerdo con Fuentes Albarrán (2009). Dentro de las características demográficas se estableció la edad de las participantes al momento de la encuesta.

\section{Para evaluar los patrones de automedicación}

Se determinó cuál es la prevalencia de automedicación con anticonceptivos en las estudiantes de la Facultad de Enfermería según la muestra, se enumeraron cuáles son los anticonceptivos orales más usados y se estableció un listado de las causas más frecuentes que llevan a la automedicación y sus manifestaciones clínicas adversas asociadas al uso de anticonceptivos sin prescripción médica. Además, se llevó a cabo la relación de algunas de las variables que nos permitieron analizar de forma más completa los resultados hallados. Los datos obtenidos se tabularon y se analizaron estadísticamente por medio del programa Microsoft Office Excel, en el cual se calcularon: promedio, media, frecuencia y porcentajes.

\section{Resultados}

Para llevar a cabo este estudio se aplicaron 230 encuestas de las cuales 100 de las estudiantes cumplieron con los criterios de inclusión (estudiantes femeninas de la Facultad de Enfermería de la Corporación Universitaria 
Remington, mujeres que usan anticonceptivos orales automedicados). Lo que representó un $43.5 \%$ de la muestra. Siendo esto significativo para el desarrollo del estudio.

\section{Característica demográfica}

\section{Edad}

La media de edad de la muestra fue de 24 años. Se dividieron los grupos etarios en cuatro: 17 a 21 años que representó un (36 \%), de 22 a 26 años (37\%), de 27 a 31 años (20\%) y de 32 a 36 años (7\%) (Figura 1).
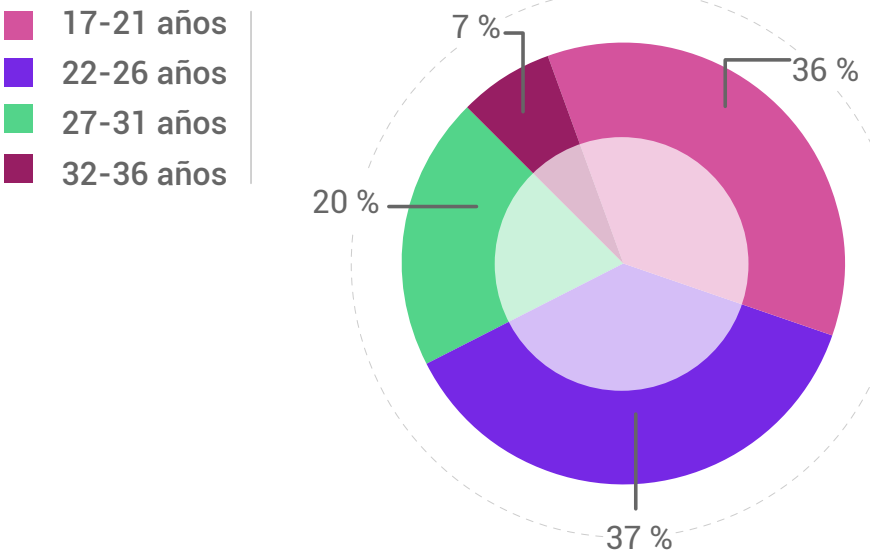

Figura 1. Distribución de la muestra de estudio de acuerdo con la edad en años.

Para realizar la división de los grupos etarios se tuvo en cuenta la desviación estándar con respecto a la media aritmética de edad de las personas encuestadas, la cual fue de 5 años. Esta distribución nos proporcionó facilidad de relacionar y analizar algunas de las variables.

\section{Patrones de automedicación}

\section{Causas de automedicación}

Según la encuesta realizada para determinar las causas que conllevan a la automedicación de anticonceptivos orales se encontró que la principal causa es la buena referencia que se tiene del medicamento con $53 \%$, seguido por que las encuestadas no les gustan los anticonceptivos que 
otorgan las Entidades Promotoras de Salud (EPS) con $17 \%$, el precio de medicamento representó un $11 \%$, y 8 \% no tienen EPS; así mismo, lo lejos que se encuentra el centro de salud representó un $6 \%$ de las causas, y el temor a que se enteren que planifica con un $4 \%$.

\section{Causas/edad}

Para las causas más prevalentes en relación con los grupos de edad se encontró que en las estudiantes de 17 a 21 años la causa principal de automedicación es la buena referencia de los anticonceptivos (61 \%). Así mismo, está fue la causa más representativa para las estudiantes de 22 a 26 años con un (51 \%) y para las de 27 a 31 años con un (55\%). Por su parte, en el grupo de 32 a 36 años la causa más prevalente fue «no me gustan los anticonceptivos de la EPS» con un 42 \% (Figura 2).

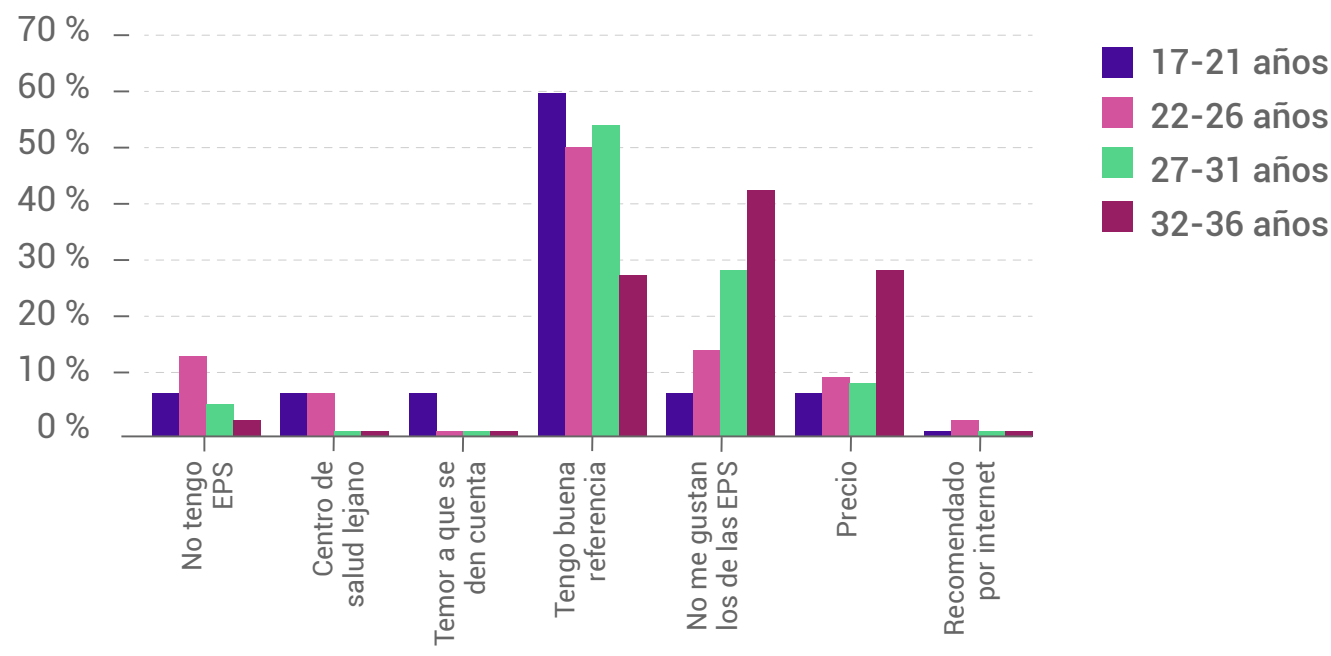

Figura 2. Causas de automedicación en relación al grupo etario.

\section{Recomendación}

La mayor parte de los encuestadas (42 \%) derivó el uso de estos medicamentos por recomendación del médico tratante al que alguna vez acudió. El $23 \%$ de recomendación de uso se acredita a enfermeras de centro de salud, el $16 \%$ uso voluntario del medicamento, $8 \%$ se basó en la recomendación de un amigo, el $6 \%$ acudió a una farmacia y el $5 \%$ menciona otros tipos de recomendaciones (televisión, internet, radio, avisos publicitarios) (Figura 3). 


\section{Recomendación}

- Receta de farmacia

- Médico tratante en el pasado

Enfermera del centro de salud

- Recomendado por un amigo

- Uso voluntario

Otros

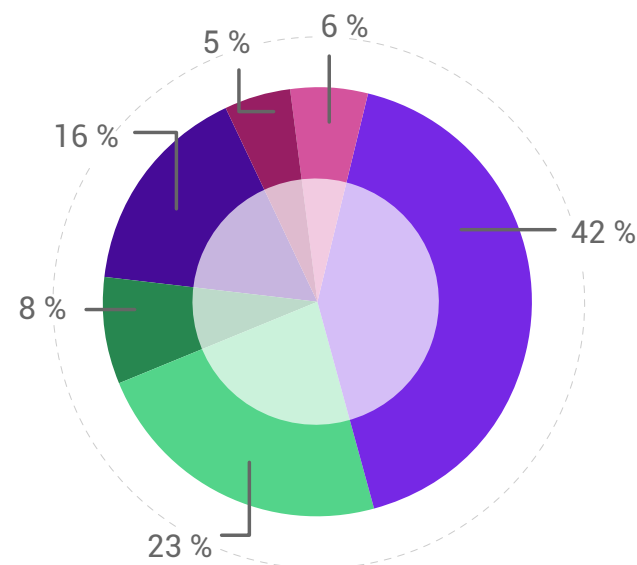

Figura 3. Recomendación del medicamento anticonceptivo.

\section{Anticonceptivos más usados}

Al establecer un listado de los medicamentos que se usan con más frecuencia en la automedicación, se encontró que los anticonceptivos orales más usados son: el Microgynon con un (19\%), el Yax (17\%), y el Diane $35(17 \%)$ (Figura 4).

Femelle 20

Gynorelle

- Yax

Noval

Lindella

- Veronia

Adelle

- Yaxibelle

- Micogynon

Diane 35

Otros: Bellaface

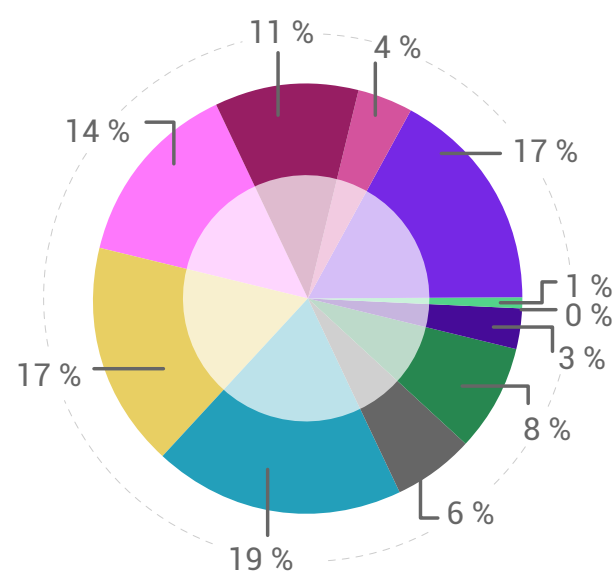

Figura 4. Porcentaje de medicamentos usados. 
De la misma manera que se usan anticonceptivos deliberadamente, también se consumen otros tipos de medicamentos. En este estudio se hace una clasificación de grupos de medicamentos que utilizan las participantes, donde los analgésicos son los de mayor uso (Figura 5).

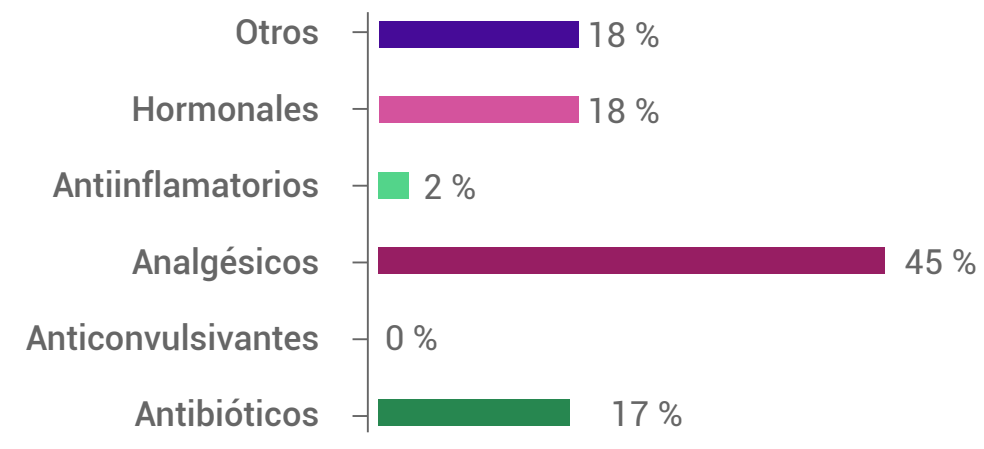

Figura 5. Medicamentos usados concomitantes con los anticonceptivos.

\section{Efectos secundarios}

El consumo de medicamentos generalmente produce efectos secundarios que puede generar problemas en la salud de quienes lo consumen. En este estudio se encontró que el efecto más frecuente asociado al uso de anticonceptivos orales fue la cefalea con un $44 \%$ (Figura 6).

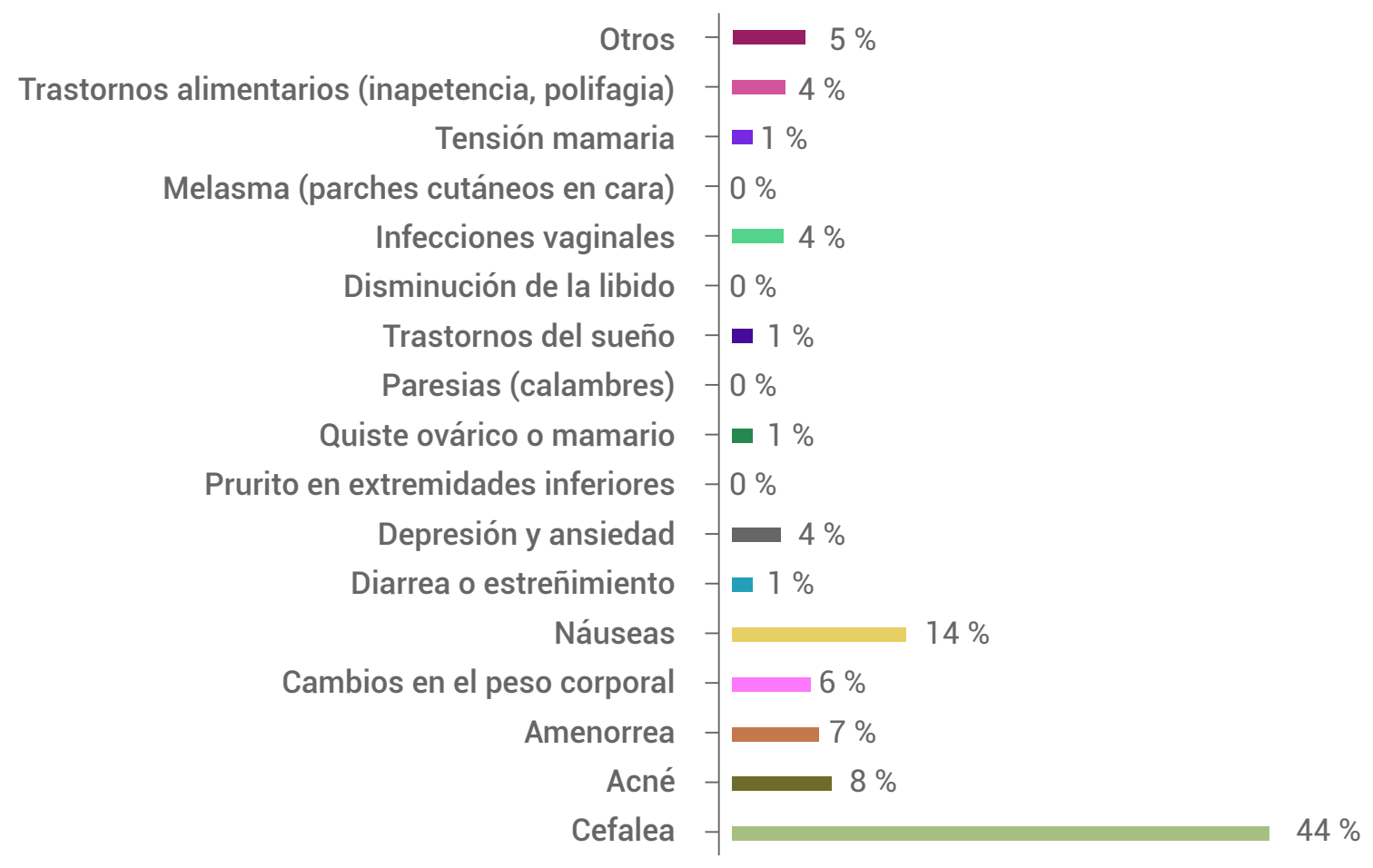

Figura 6. Efectos adversos frecuentes. 
Con relación a los anticonceptivos más usados y a los efectos secundarios que más se presentaron en las estudiantes quienes lo consumen fue cefalea: Microgynon en un (63\%), con el Yax en un (41 \%), y con el Diane 35 (41 \%) (Figura 7).

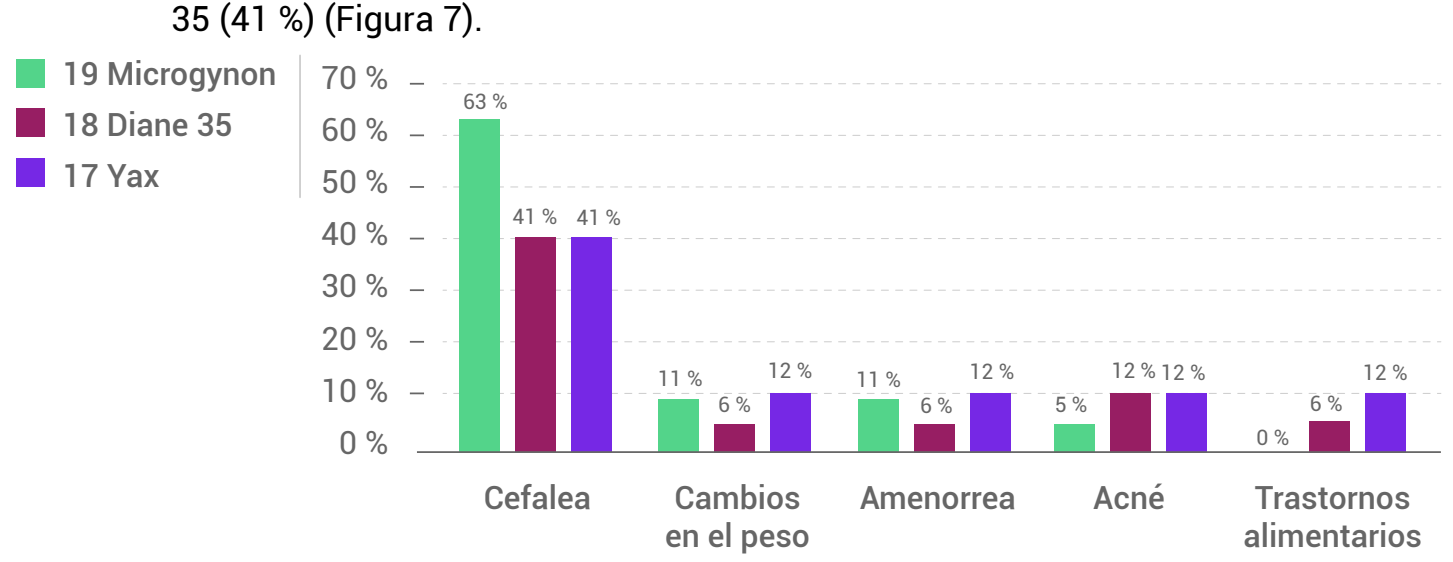

Figura 7. Porcentajes de efectos adversos con relación a medicamentos de mayor uso.

\section{Tiempo de uso de medicamentos}

La determinación del tiempo que las participantes llevan usando el medicamento de anticoncepción oral es importante para la asociación de esto con algunos de los posibles efectos adversos. Se halló que la mayor parte de las participantes lleva consumiendo de 1-3 años los fármacos, lo que representó un $42 \%$, siguiendo menos de 1 año de uso con el $39 \%$; un $15 \%$ respondió que usa el anticonceptivo de $4-6$ años, y el $4 \%$ dice llevar más de 6 años con el método.

\section{Discusión}

La automedicación con anticonceptivos orales, en la Facultad de Enfermería de la Corporación Universitaria Remington, es un fenómeno latente, que se evidencia a través de la realización de este estudio que arrojó:que de la muestra utilizada el $43,5 \%$ se automedica este tipo de fármaco. Lo que nos permite relacionar con el estudio realizado en la Facultad de Enfermería de la Universidad Federal de Goiás, el cual mostró que la prevalencia de estudiantes que practicaban la automedicación fue de 38.8 \% (Ferreira Souza L., Damázio da Silva C., Carvalho Ferraz G., Faleiros Sousa F. y Varanda Pereira, 2011). También con el estudio hecho en la Universidad Complutense de Madrid sobre la automedicación de fármacos en la población estudiantil, muestra cómo los alumnos de la rama de ciencias de la salud, representan el mayor porcentaje de los que se automedican: $5 .^{\circ}$ curso $(60 \%)$, seguido de $4 .^{\circ}(70.37 \%), 3 .^{\circ}$ (75\%), $2^{\circ}(66.67 \%)$ y $1 .^{\circ}(65.22 \%)$ (Garcés Ranilla y Delgado Serrano, 
2014). Lo anterior pone al descubierto que los estudiantes del área de la salud son una de la población con gran índice de prácticas de automedicación haciéndonos cuestionar, sobre si el hecho de formarse como personal de salud tiene influencia en este fenómeno.

Dentro los hallazgos en nuestro estudio la automedicación predomina en la edad de los 22 a 26 años, se relaciona con un estudio llevado a cabo en la Universidad de Antioquia para determinar el uso de fármacos anticonceptivos orales donde se incluyeron 353 mujeres y la edad promedio fue de 21.3 años. Demostrando como las participantes en esta edad hacen efectivo el uso de estos medicamentos por iniciativa propia. Además, el mismo estudio mostró que el $24.7 \%$ de las usuarias toman o tomaron por autoprescripción (Hincapié García, et al., 2013)

Puesto que un gran porcentaje de las estudiantes que participaron en el estudio derivó su acceso a la automedicación por una recomendación médica del pasado ( $42 \%$ ), cabe resaltar lo mucho que influye una prescripción antigua en el consumo de este tipo de medicamento, no teniendo en cuenta que para el uso frecuente y prolongado de este grupo de fármaco se requiere una revisión periódica del estado fisiológico de las usuaria, lo que proporcionara el grado de efectividad y la aparición de algunos efectos no deseables. Lo que hace pensar en lo oportuno de generar nuevos estudios en cuanto a la periodicidad con que las usuarias de los anticonceptivos orales deberían realizar chequeos generales y el importante papel del personal de enfermería en la elección y uso de estos medicamentos.

Al momento de determinar las causas principales que conllevan a la automedicación, se halló en este estudio como factor causal: la buena referencia que se tiene del medicamento que se consume. Este resultado se puede asociar con el estudio que se realizó en Perú en donde se encontró que una de las principales causas para automedicarse fue el «conocimiento suficiente» sobre el medicamento (Llanos, Contreras, Velásquez, Mayca, Lecca, Reyes y Peinado, 2001). Lo que destaca que la información sobre los medicamentos que usan es un determinante que lleva a esta práctica. En muchas de los casos no siempre es suficiente el hecho de creer que se tiene el conocimiento $y$, más aún tratándose del consumo de este tipo de medicamentos que si se usan de manera no propicia y hay presente hábitos inapropiados para la salud, se verá afectada su eficacia y los efectos no deseados subyacentes en el cuerpo tendrán mayor riesgo de aparecer. Asimismo, como se evidenció en el estudio hecho en Sevilla - España, con el fin de determinar el conocimiento sobre el uso de anticonceptivos orales que demostró que, pese a que las mujeres encuestadas poseen un alto conocimiento sobre la utilización de anticonceptivos orales, la mitad de la población sometida a análisis reconoció ser fumadora, aunque estuviera contraindicado en usuarias de este medicamento. Suponiendo que es un factor que aumenta el riesgo cardiovascular y las posibilidades de sufrir tromboembolismo venoso (Ferrer, Murillo y Machuca, 2003).

Además de evidenciar la automedicación de anticonceptivos orales, hallamos que una gran parte de las encuestadas usa de manera concomitante analgésicos. Lo que se puede asociar con un estudio llevado en una universidad de Valencia - España, donde el grupo de analgésicos fueron los fármacos que más se automedicaron los estudiantes participantes (Guillem Sáiza, Francés Bozala, Gimenez Fernández y Saiz Sánchez, 2010). Es importante resaltar 
que la cefalea es uno de los efectos secundarios que más se presenta en las estudiantes que consumen los anticonceptivos orales, de allí parte la relación del hallazgo de los analgésicos como fármacos usados de manera reiterativa. Sabemos que el uso de fármacos al mismo tiempo puede causar interacciones que modifican el mecanismo de acción de estos, en este caso el uso de analgésicos como paracetamol (acetaminofén), salicilatos (ASA); vemos como los componentes estrogénicos de los anticonceptivos orales actúan como inductores hepáticos y reducen la intensidad y duración del efecto analgésico del paracetamol, también disminuye la concentración de acetil salicílico, lo que hace factible en algunos casos el aumento de la dosis de analgésicos (Pérez Landin, 2008).

Caso contrario con otros analgésicos que contienen fenacetina y pirazolona. La clorpromacina, la dihidroergotamina y las benzodiacepinas han estado asociadas con la reducción de la eficacia anticonceptiva y una mayor frecuencia de sangrado por deprivación no esperado (Torres Serna, 2006).

Al determinar los medicamentos anticonceptivos más usados por las estudiantes, se encontró que fueron el Microgynon, Yax, y Diane 35. Esto llama la atención porque el estudio de seguimiento farmacoterapeútico en estudiantes universitarias sobre el consumo y conocimientos de anticonceptivos orales, menciona que los anticonceptivos más consumidos son el Diane 35 y el Yax, ya que son de fácil acceso (Sánchez del Moral y Herrera Carranza, 2005)

Se hace una relación entre los medicamentos más usados y los efectos secundarios, en la que se encuentra que la cefalea es el efecto que prevalece dentro estos medicamentos. Caso contrario a lo que se demostró en el estudio donde se comparan los efectos adversos presentes entre dos anticonceptivos; entre las reacciones adversas más referidas por las féminas sobresalieron los trastornos gastrointestinales con 81 pacientes, para $40.5 \%$, predominantemente las náuseas y la epigastralgia (39\%), los que fueron notificados por $46.6 \%$ y $35 \%$ de las consumidoras de Triquilar y Microgynon, respectivamente. La ganancia de peso fue el segundo efecto en orden de frecuencia, causada por el Microgynon (70 \%) y el Triquilar (40\%). Lo que demuestra la poca relación del Microgynon con la aparición de cefalea (Arrate Negret, Linares Despaigne, Molina Hechavarría, Sánchez Imbertl y Arias Carbonell, 2013).

De acuerdo con el tiempo de uso de los anticonceptivos orales, llama la atención el hecho de que las estudiantes lleven usando ese tipo de fármacos en un periodo de 1 a 3 años, siendo un $42 \%$ de las participantes, ya que se ha relacionado el tiempo prolongado de uso con aparición de algunas enfermedades. Un artículo de revisión buscó establecer la aparición de tromboembolismo venoso y los anticonceptivos orales en donde según sus conclusiones no hay acuerdo definitivo en la literatura científica sobre si las diferentes progestinas usadas en los anticonceptivos orales y el riesgo de tromboembolismo venoso de cada una, tengan un impacto clínico significativo. Aun si los anticonceptivos de 3. ${ }^{\mathrm{a}}$ y $4 .^{\mathrm{a}}$ generación se han asociado a mayor riesgo relativo, la diferencia absoluta en riesgo es pequeña, ha sido estimada por diversos autores de 4-6 casos atribuibles por 10000 usuarias por año. Para reducir el riesgo de tromboembolismo venoso, es importante evitar la prescripción de anticonceptivos orales en mujeres de riesgo elevado. El uso de una herramienta como «Los Criterios Médicos de Elegibilidad de la OMS», constituye 
una de las principales guías para los prescriptores (Pizzi Laveglia, 2014).

\section{Conclusión}

Los hallazgos del presente trabajo son de gran importancia para nuestra comunidad universitaria y como enfermeros profesionales en formación, ya que se puede evidenciar la alta incidencia de automedicación de anticonceptivos orales en las estudiantes de enfermería de la Corporación Universitaria Remington. Aunque la causa de la automedicación más frecuente fue la buena referencia del fármaco, es propicio generar conciencia sobre lo importante de una asesoría correcta y que cumpla los parámetros de seguridad y efectividad con respecto al uso de los anticonceptivos orales.

Si bien las reacciones adversas con relación a los fármacos usados encontradas en el estudio no muestran coherencia con estudios anteriores que demuestran que los efectos colaterales más importantes son aumento de peso, náuseas y epigastralgia, en nuestra población de estudio se evidencia la cefalea como el más frecuente. Se generan opiniones críticas en cuanto a los anticonceptivos orales más consumidos, porque a pesar de causar diferentes efectos secundarios de acuerdo a cada mujer que lo usa, son eventos que afectarán la salud y calidad de vida. De allí la gran importancia de un adecuado seguimiento y educación para la adquisición del método.

Sabiendo que el uso concomitante de los anticonceptivos orales con los analgésicos (acetaminofén, aspirina) no muestra disminución de la efectividad del anticonceptivo, existe la excepción con otros analgésicos los cuales se asocian a una reducción del mecanismo acción de los anticonceptivos. Esto expone los posibles problemas que representa el consumo de distintos fármacos al mismo tiempo, dado que una interacción medicamentosa no adecuada disminuirá la efectividad de estos y, más aún, el caso de los anticonceptivos orales que, como consecuencia de disminuir su efecto, existe el riesgo de un embarazo no deseado.

\section{Referencias}

- Álvarez, J. (2013). Prevalencia de automedicación en estudiantes de farmacia y medicina de la universidad de granada. Tesis de grado en máster de seguimiento farmacéutico terapéutico. Universidad de granada, Facultad de Farmacia. 1: 1-42.

Arrate, M.M., Linares, M.J., Molina, V., Sánchez, N. y Arias, M.M. (2013). Efectos secundarios de los anticonceptivos hormonales en usuarias del método asistentes a las consultas de planificación familiar. MEDISAN, 17(3):415-425.

- Cáceres, E., Thornberry, J., Má Cárdenas, L., y Ramos, J. (2014). Proyecto PIENSA (Programa de Investigación y Educación en Salud Reproductiva): impacto de la asesoría de la elección anticonceptiva en las mujeres peruanas. Rev. Peruana de Ginecología y Obstetricia, 60(3): 227-232.

- Ferreira, L., Damázio, C., Carvalho, G., Faleiros, F. y Varanda, L. (2011). Prevalencia y caracterización de la práctica de automedicación para alivio del dolor entre estudiantes universitarios de enfermería. Rev. Latino-Am. Enfermagem, 19(2): 1-7.

- Ferrer, I., Murillo, M.D. y Machuca, M., (2003). Determinación del grado de conocimiento y correcta utilización sobre anticonceptivos orales en farmacia comunitaria. Seguim Farmacote, 1(3): 136-138. 
- Fuentes, K.A. (2006). Análisis y cuantificación de los patrones de automedicación en usuarios de farmacias SalcoBrand de Valdivia. Tesis de grado para título de Químico Farmacéutico. Escuela de Química y Farmacia, Facultad de Ciencias. Universidad Austral de Chile.

- Garcés, B. y Delgado, L. (2014) Estudio de consumo de fármacos en universitarios. Rev. Reduca, Serie congreso de Alumnos, 6(1): 1-10.

- Guillem, P., Francés, F., Gimenez, F. y Saiz, C. (2010). Estudio sobre Automedicación en Población Universitaria Española. Rev CLÍN MED FAM. 3(2): 99-103.

- Hincapié, J.A., Quintero, M., Gaviria, J., Estupiñán, H. y Amariles, P. (2013). Causas de abandono, cambio o fallo terapéutico de la anticoncepción hormonal en mujeres universitarias. Rev. CES Med, 27(2),153-162.

- Llanos, L.F., Contreras, C.E., Velásquez, J.E., Mayca, J.A., Lecca, L., Reyes, R. y Peinado, J. (2001). Automedicación en cinco provincias de Cajamarca, Perú. Rev Med Hered, 12: 127-133.
Pérez, B. (2008). Interacciones de los anticonceptivos orales. Rev. AULAdelafarmacia, $48 ;-51$.

- Pizzi, R. (2014). Anticonceptivos orales y tromboembolismo venoso: la controversia de los progestágenos. Rev Obstet Ginecol Venez, 74(3):145-147.

- Sánchez, R. y Herrera, J. (2005). Conocimiento de los medicamentos anticonceptivos en una población universitaria. Seguimiento Farmacoterapéutico, 3(3): 130-134.

- Torres, C. (2006). Anticoncepción en situaciones especiales: Anticonceptivos hormonales y su interacción con otros medicamentos. (24 ed.) Bogotá, Colombia: Distribuna editorial médica. 